\title{
Facial dermoid cyst
}

INSERM

\section{Source}

INSERM. (1999). Orphanet: an online rare disease and orphan drug data base. Facial dermoid cyst. ORPHA:141051

Facial dermoid cyst is a rare, benign cutaneous neoplasm containing keratinized epithelium and dermal derivatives, such as hair follicles, sweat and sebaceous glands, smooth muscle or fibroadipose tissue, which usually manifests as a firm, nonpulsatile mass, often with a sinus opening or a hair-bearing punctum, most commonly located in the periorbital and nasal area. 